Research Article

\title{
Mechanism of Cordyceps Cicadae in Treating Diabetic Nephropathy Based on Network Pharmacology and Molecular Docking Analysis
}

\author{
Yi Qian, ${ }^{1}$ Xin Sun, ${ }^{1}$ Xin Wang, ${ }^{1}$ Xin Yang, ${ }^{1}$ Mengyao Fan, ${ }^{1}$ Jiao Zhong, ${ }^{1}$ Zejun Pei $\mathbb{D},{ }^{1}$ \\ and Junping Guo $\mathbb{D}^{2}$ \\ ${ }^{1}$ Department of Pharmacy, The Affiliated Wuxi No. 2 People's Hospital of Nanjing Medical University, Wuxi 214002, China \\ ${ }^{2}$ Yixing People's Hospital, Yixing 214200, China
}

Correspondence should be addressed to Zejun Pei; pei-zj@126.com and Junping Guo; staff187@yxph.com

Received 16 July 2021; Revised 25 August 2021; Accepted 8 September 2021; Published 28 September 2021

Academic Editor: Liping Yu

Copyright (C) 2021 Yi Qian et al. This is an open access article distributed under the Creative Commons Attribution License, which permits unrestricted use, distribution, and reproduction in any medium, provided the original work is properly cited.

Objective. To systematically study the mechanism of cordyceps cicadae in the treatment of diabetic nephropathy (DN) with the method of network pharmacology and molecular docking analysis, so as to provide theoretical basis for the development of new drugs for the treatment of DN. Methods. TCMSP, Symmap, PubChem, PubMed, and CTD database were used to predict and screen the active components and therapeutic targets for DN. The network of active components and targets was drawn by Cytoscape 3.6.0, the protein-protein interaction (PPI) was analyzed by the STRING database, and the DAVID database was used for the enrichment analysis of intersection targets. Molecular docking studies were finished by Discovery Studio 3.5. Results. A total of 36 active compounds, including myriocin, guanosine, and inosine, and 378 potential targets of cordyceps cicadae were obtained. PPI network analysis showed that AKT1, MAPK8, and TP53 and other targets were related to both cordyceps cicadae and DN. GO and KEGG pathway analysis showed that these targets were mostly involved in R-HSA450341, 157.14-3-3 cell cycle, and PDGF pathways. Docking studies suggested that myriocin can fit in the binding pocket of two target proteins (AKT1 and MAPK8). Conclusion. Active ingredients of cordyceps cicadae such as myriocin may act on DN through different targets such as AKT1, MAPK8, and TP53 and other targets, which can help to develop innovative drugs for effective treatment of DN.

\section{Introduction}

Diabetic nephropathy (DN), also known as diabetic kidney disease (DKD), is one of the most common secondary nephrosis with high incidence rate and low cure rate. DKD develops in approximately $40 \%$ of patients who are diabetic and is the leading cause of chronic kidney disease (CKD) worldwide [1]. It increases the death rate in diabetic patients [2]. At present, there are effective approaches that can reduce the incidence of diabetic kidney disease and postpone its progression such as controlling blood glucose levels and blood pressure as well as blockade of the renin-angiotensinaldosterone system [3]. Many researches of traditional Chinese medicine have also been carried out in the treatment of DN and have made some achievement in recent years, such as berberine [4], Qidan Dihuang grain (QDDHG) [5], and Danggui Shaoyao San (DSS) [6]. The mechanism of action may be multifaceted, such as regulating glucose metabolism, correcting lipid metabolism disorder, inhibiting the activation of polyol pathway, antioxidative stress, improving the structure and function of podocytes, inhibiting inflammatory response, and intervening cell signal transduction. However, there remains an urgent need for innovative drugs to treat $\mathrm{DN}$.

Cordyceps cicadae, also called "Chan Hua," belongs to the family Clavicipitaceae, Ascomycotina, and its anamorph is Isaria cicadae Miq. Furthermore, cordyceps cicadae has been used as a substitute for cordyceps sinensis [7]. Cordyceps cicadae is one of the most famous traditional Chinese medicines and has been used for about 1600 years in China. 


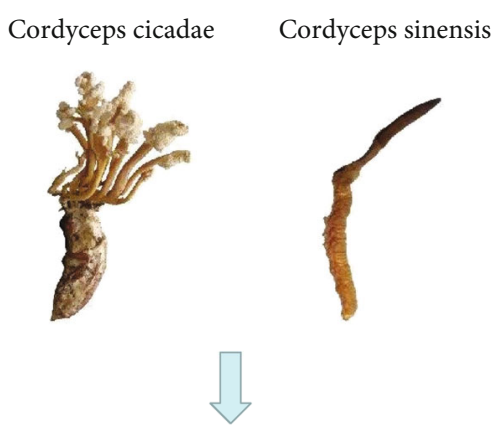

TCMSP, PubChem, Symmap, PubMed

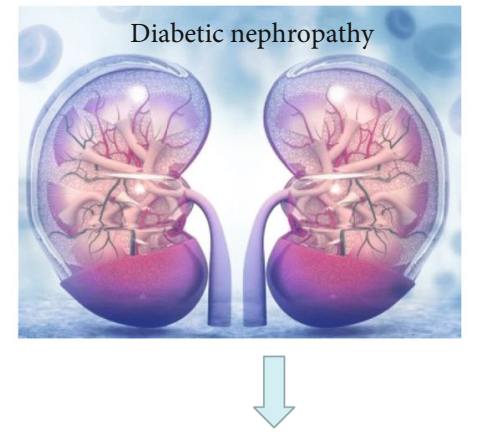

CTD database
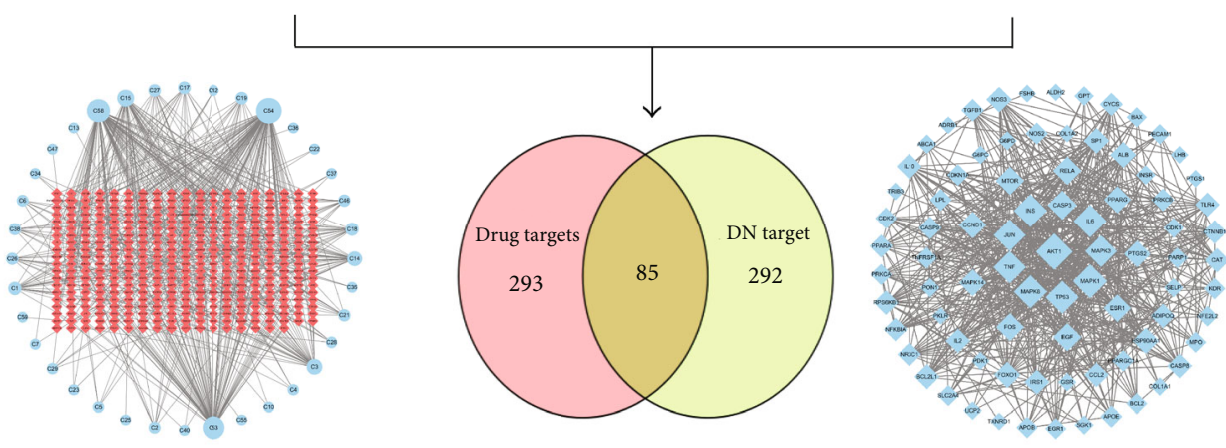

PPI analysis

Network construction

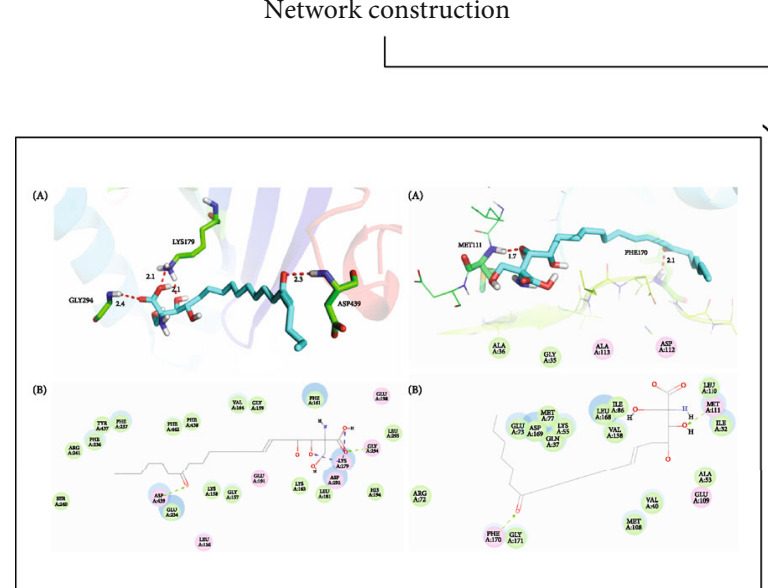

Molecular docking

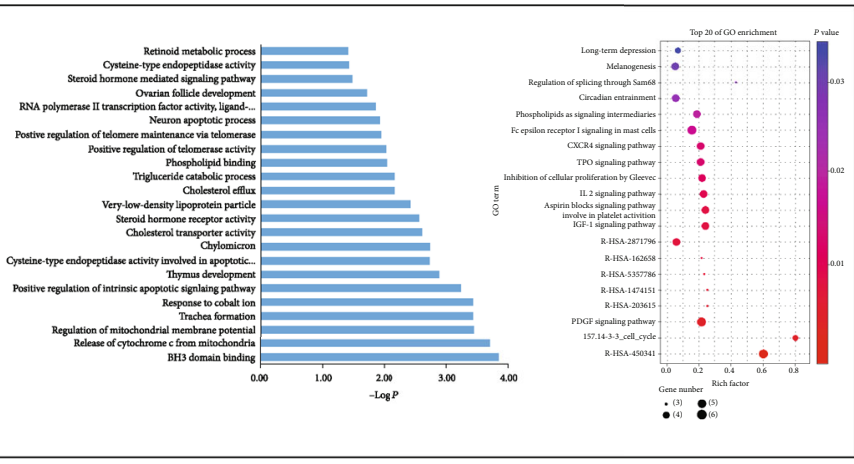

GO and KEGG enrichment analysis

FIGURE 1: Network pharmacology workflow of cordyceps cicadae and diabetic nephropathy.

Cordyceps cicadae is a kind of cordyceps fungus produced by Paecilomyces sp. after infecting cicada. It belongs to the same insect fungus complex as cordyceps sinensis. Li's research has suggested that HEA, one active component of cordyceps cicadae, could alleviate many diabetes complications in genetically obese mice and may offer promise as a supplement for diabetes management [8]. Another study showed that cordyceps cicadae had the antidiabetic activity in a diabetic rat model and could be a promising therapeutic source in managing diabetes mellitus and its associated complications [9]. It was reported that the results indicated that CCP, one important component of cordyceps cicadae, improved insulin resistance and glucose tolerance in DN rats. Furthermore, CCP intervention significantly suppressed the inflammation, renal pathological changes, and renal dysfunction, slowing down the progression of renal interstitial fibrosis [10].

Network pharmacology, which is aimed at studying the complex, diverse relationships between targets, drugs, diseases, and pathways, presents a new approach for drug discovery [11]. Network pharmacology explores the relationship between traditional Chinese medicine and disease at the overall level, which provides a simple way to explain the mechanism of traditional Chinese medicine treatment. Network pharmacology uses bioinformatics to help us get a better understanding of drug actions and thereby to advance drug discovery.

This study is aimed at exploring the therapeutic effects and mechanisms of cordyceps cicadae on DN by the network pharmacology approach. The workflow is shown in Figure 1. 
TABle 1: Candidate compounds of cordyceps cicadae.

\begin{tabular}{|c|c|c|c|c|c|}
\hline No. & Name of compounds & $\begin{array}{c}\text { Number of } \\
\text { targets }\end{array}$ & No. & Name of compounds & $\begin{array}{c}\text { Number of } \\
\text { targets }\end{array}$ \\
\hline $\mathrm{C} 1$ & Oleic acid & 24 & $\mathrm{C} 32$ & Cerevisterol & 0 \\
\hline $\mathrm{C} 2$ & EIC & 15 & $\mathrm{C} 33$ & Cholesteryl palmitate & 0 \\
\hline $\mathrm{C} 3$ & Arachidonic acid & 38 & $\mathrm{C} 34$ & (2R,3S,5S)-5-(6-Aminopurin-9-yl)-2-(hydroxymethyl)oxolan-3-ol & 3 \\
\hline $\mathrm{C} 4$ & Linoleyl acetate & 4 & $\mathrm{C} 35$ & (2R,3R,4S)-2-(6-Aminopurin-9-yl)-4-(hydroxymethyl)oxolan-3-ol & 3 \\
\hline $\mathrm{C} 5$ & Vitamin C & 6 & $\mathrm{C} 36$ & Cordylagenin & 1 \\
\hline C6 & Uracil & 13 & $\mathrm{C} 37$ & CLR & 4 \\
\hline $\mathrm{C} 7$ & Adenine & 4 & $\mathrm{C} 38$ & Cinnamaldehyde & 12 \\
\hline $\mathrm{C} 8$ & Styrone & 0 & C39 & Ignavine & 0 \\
\hline C9 & 20-Hexadecanoylingenol & 0 & $\mathrm{C} 40$ & Deoxyandrographolide & 3 \\
\hline $\mathrm{C} 10$ & Vitamin G & 2 & $\mathrm{C} 41$ & Karakoline & 0 \\
\hline C11 & D-Mannoheptulose & 0 & $\mathrm{C} 42$ & Isotalatizidine & 0 \\
\hline $\mathrm{C} 12$ & Ergosterol & 5 & $\mathrm{C} 43$ & Neokadsuranic acid A & 0 \\
\hline $\mathrm{C} 13$ & MTL & 2 & $\mathrm{C} 44$ & 2,7-Dideacetyl-2,7-dibenzoyl-taxayunnanine F & 0 \\
\hline $\mathrm{C} 14$ & Beta-sitosterol & 38 & $\mathrm{C} 45$ & 3-Acetylaconitine & 0 \\
\hline $\mathrm{C} 15$ & Caffeine & 54 & $\mathrm{C} 46$ & Berberine & 12 \\
\hline $\mathrm{C} 16$ & TGL & 0 & $\mathrm{C} 47$ & Neokadsuranic acid C & 1 \\
\hline $\mathrm{C} 17$ & Nicotinic acid & 17 & $\mathrm{C} 48$ & Hypaconitine & 0 \\
\hline $\mathrm{C} 18$ & GLB & 34 & $\mathrm{C} 49$ & Deoxyaconitine & 0 \\
\hline $\mathrm{C} 19$ & Uralene & 15 & C50 & Adenosine & 0 \\
\hline $\mathrm{C} 20$ & TRE & 0 & C51 & N-(2-Hydroxyethyl)adenosine & 0 \\
\hline $\mathrm{C} 21$ & GUP & 23 & C52 & $\begin{array}{c}\text { 1-[(2R,3R,4S,5S)-3,4-Dihydroxy-5-(hydroxymethyl)oxolan-2- } \\
\text { yl]pyrimidine-2,4-dione }\end{array}$ & 0 \\
\hline $\mathrm{C} 22$ & Uridine & 2 & C53 & Inosine & 89 \\
\hline $\mathrm{C} 23$ & Thiamine & 4 & $\mathrm{C} 54$ & Guanosine & 107 \\
\hline $\mathrm{C} 24$ & Peroxyergosterol & 0 & C55 & GUN & 1 \\
\hline $\mathrm{C} 25$ & Galactomannan & 1 & C56 & Beauverin & 0 \\
\hline $\mathrm{C} 26$ & Palmitic acid & 17 & $\mathrm{C} 57$ & $\mathrm{~N}$-(4-Aminobenzoyl)-L-glutamic acid & 0 \\
\hline $\mathrm{C} 27$ & Linoleic & 14 & C58 & Myriocin & 89 \\
\hline $\mathrm{C} 28$ & NCA & 7 & C59 & Ergosta-4,6,8(14),22-tetraene-3-one & 1 \\
\hline C29 & Stearic acid & 7 & $\mathrm{C} 60$ & Hyaluronic acid & 0 \\
\hline $\mathrm{C} 30$ & LFA & 0 & C61 & 5,6-Epoxyergosta-7,22-dien-3-ol & 0 \\
\hline C31 & Isoergotamine & 0 & & & \\
\hline
\end{tabular}

\section{Materials and Methods}

2.1. Identification of Candidate Components. Most components are collected from the Traditional Chinese Medicine Systems Pharmacology Database and Analysis Platform (TCMSP) database (http://tcmspw.com/tcmsp.php). On account of that, cordyceps cicadae contains almost all the same active components of cordyceps sinensis; the keywords are "Cordyceps sinensis" and "cordyceps cicadae" so that the eligible active components and corresponding targets are collected. Symmap database (http://www.symmap.org/) was used to search all the active components and targets with the keywords of "Cordyceps sinensis" and "cordyceps cicadae." The compounds and corresponding targets with $\mathrm{OB}$ $\geq 30 \%$ were screened out, and the repetitive compounds were removed. Moreover, PubMed (https://pubmed.ncbi .nlm.nih.gov) was used to search the literature for further information. Finally, the candidate active compounds were identified.

2.2. Prediction Targets of Candidate Active Compounds. The targets were obtained from four aspects: (1) PubChem database (http://pubchem.ncbi.nlm.nih.gov), (2) TCMSP database, (3) Symmap database, and (4) PubMed database.

2.3. Prediction Targets of Diabetic Nephropathy. With "diabetic nephropathy" as keywords, the CTD database was used (http://ctdbase.org/about/) to search genes related to diabetic nephropathy.

2.4. Network Construction. The active component target network of cordyceps cicadae was constructed by using the software Cytoscape 3.6.0 to analyze the association between 


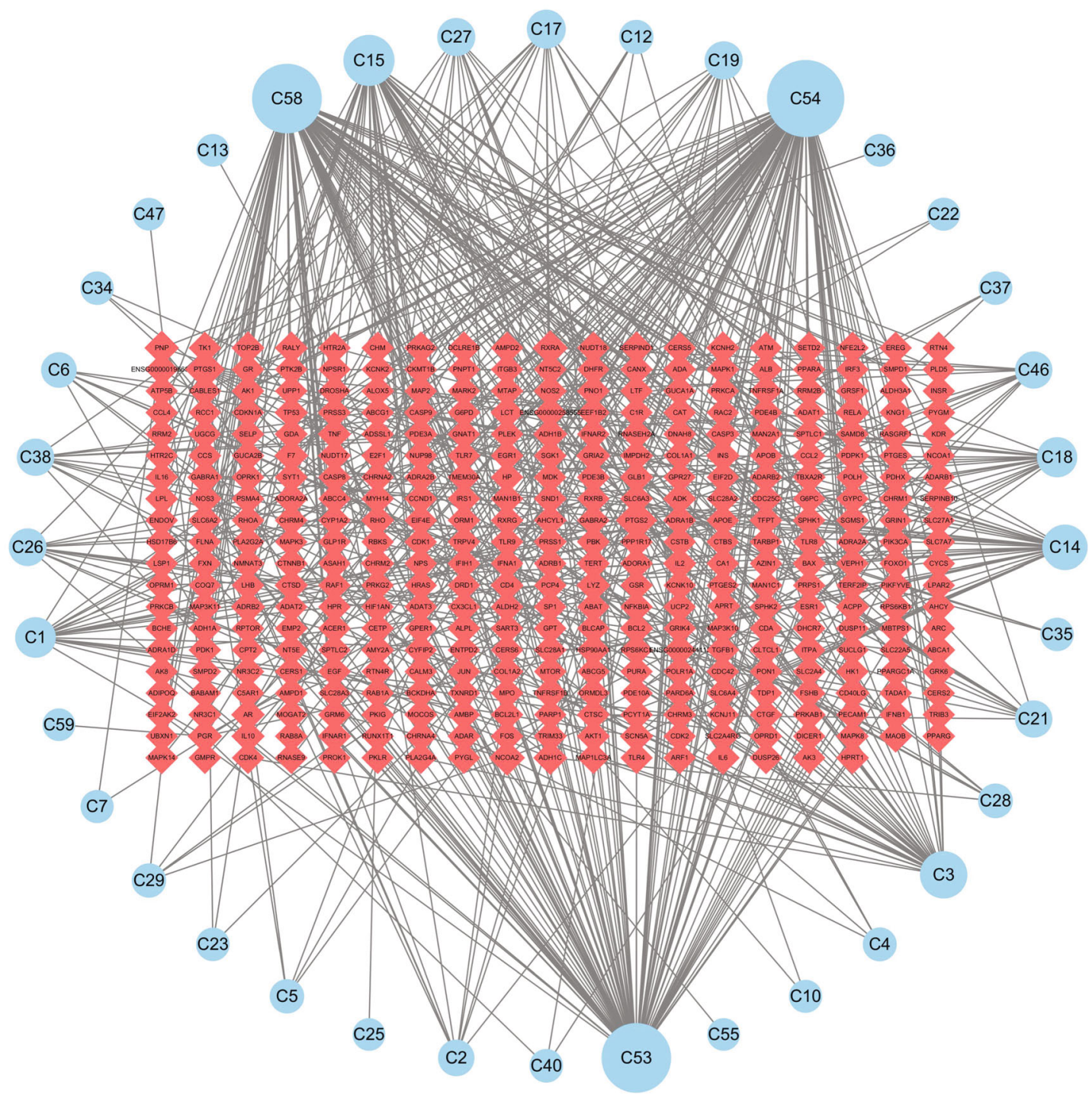

FIgURE 2: The active components and target network of cordyceps cicadae. The blue circle represents the component of cordyceps cicadae; the red diamond represents the target; the size of the node represents the size of the node degree.

components and targets, and the degree between candidate compounds and targets was analyzed.

2.5. Protein-Protein Interaction (PPI) Network Construction. The intersection targets of candidate compound and diabetic nephropathy were obtained by using the omicshare website (http://www.omicshare.com/). The intersection targets of candidate compound and diabetic nephropathy were imported into the STRING database (https://string$\mathrm{db} . \mathrm{org} /$ ) to obtain protein interaction information. The condition was limited to "Homo sapiens." The confidence score with correlation degree was set to $\geq 0.700$. The PPI data was imported into the software Cytoscape 3.6.0 to construct the network diagram of intersection target protein interaction.

2.6. GO and KEGG Enrichment Analysis for Targets. In order to further analyze the function of the selected targets and their role in the signal pathway, the intersection targets of the active components and diabetic nephropathy were uploaded to the DAVID database for biological process enrichment analysis and pathway enrichment analysis.

2.7. Prediction of Binding Modes between Myriocin and Candidate Two Target Proteins. Docking studies were finished by Discovery Studio 3.5 to explore the predicted binding modes of myriocin in AKT1 (PDB code: 3OCB) and MAPK8 (PDB code: 4G1W), respectively, which were downloaded from RCSB Protein Data Bank (http:// www.pdb.org/). The proteins were prepared using the Prepare Protein protocol in DS3.5, to remove all crystallographic water, add hydrogen atoms, repair broken chains, and add CHARMm force field. A sphere binding site was generated using the define site tool in DS3.5. Before docking, the small molecule myriocin was prepared using the Prepare or Filter Ligands protocol in DS3.5. The CDOCKER protocol in DS3.5 was used to perform the molecular docking. The images were created by PyMOL. 


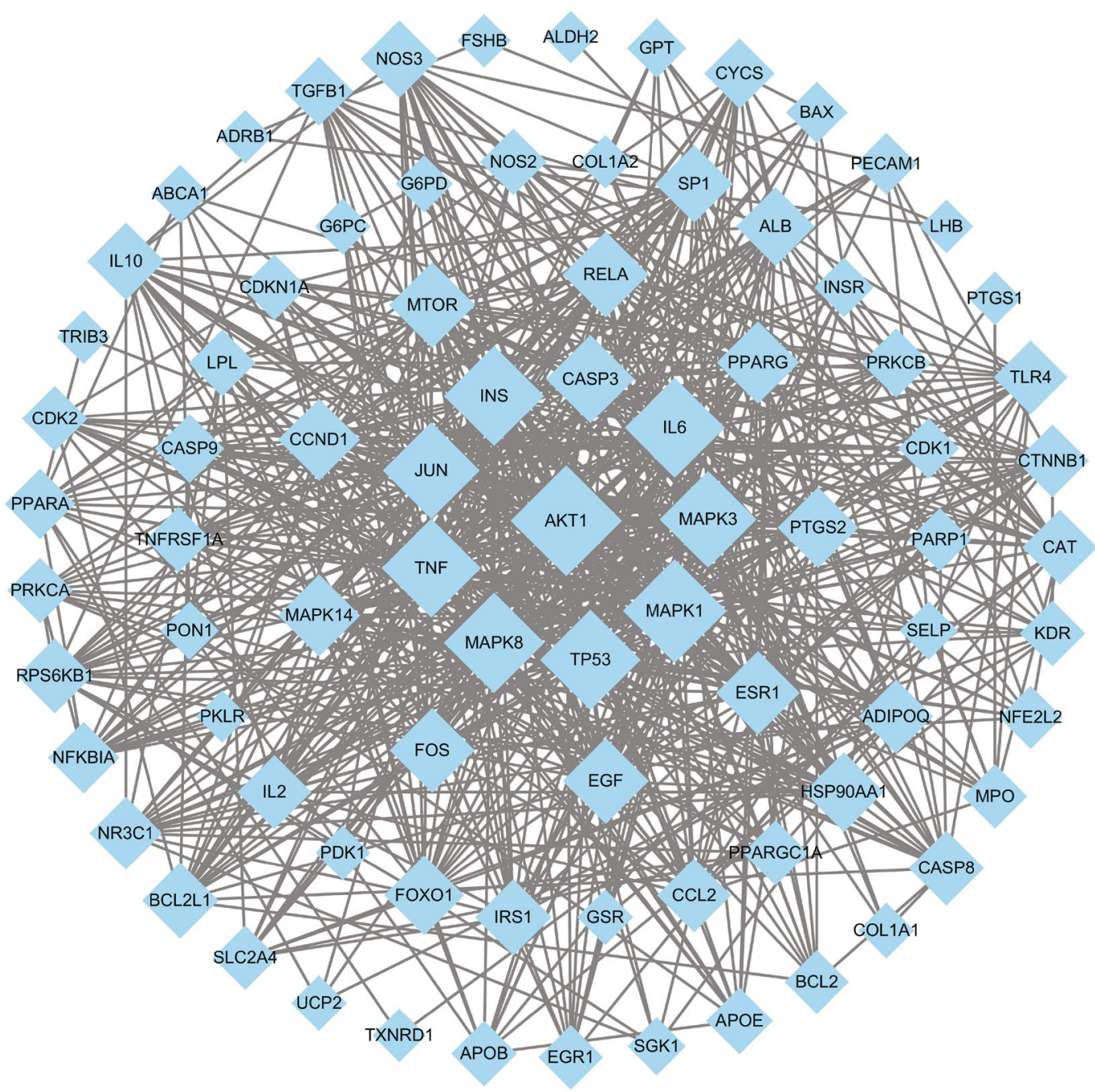

FIgURE 3: Target-diabetic nephropathy protein-protein interaction (PPI) network. AKT1, MAPK8, TP53, INS, MAPK1, IL6, TNF, JUN, MAPK3, and CASP3 were the targets with higher degree $(>30)$.

\section{Results}

3.1. Screening of Bioactive Compounds from Cordyceps Cicadae. 38 compounds of cordyceps sinensis were found by TCMSP database. 31 compounds of cordyceps sinensis were screened by Symmap database with $\mathrm{OB} \geq 30 \% .12$ active compounds of cordyceps cicadae were found by literature. A total of 61 candidate compounds were found. According to these methods, 378 targets related to candidate compounds were found (Table 1).

3.2. Targets and Active Compounds of Cordyceps Cicadae Network Construction. Targets related to the candidate compounds were obtained by using the above methods, the components and targets were imported into the Cytoscape 3.6.0 software, and the network of active compounds and targets of cordyceps cicadae was obtained (shown in Figure 2). A total of 61 compounds were screened out, of which 25 compounds were not found corresponding targets in the database. Therefore, Figure 2 only showed the interaction network between 36 compounds and their related targets.
The blue circle represented the candidate compounds, and the red diamond represented the targets. The more edges connecting to the node were, the higher the degree value of the node was. In this network, guanosine (C54) had 107 targets, myriocin (C58) had 89 targets, and inosine (C53) had 89 targets. Guanosine and inosine were the common components of cordyceps sinensis and cordyceps cicadae, and myriocin was the unique component of cordyceps cicadae. These multitarget compounds might be the core components of cordyceps cicadae. PTGS2 (prostaglandin endoperoxidase synthase 2) and PTGS1 (prostaglandin endoperoxidase synthase 1) were the targets with high degree value in the network, respectively. The targets with higher degree value might be the key targets for the efficacy of cordyceps sinensis and cordyceps cicadae.

3.3. Target-DN PPI Network. 377 genes related to DN were obtained in the CTD database with the inference score $>40$. The 378 targets of compounds were intersected with 377 targets related to $\mathrm{DN}$, and 85 important targets were obtained. These 85 targets were uploaded to the STRING database. 


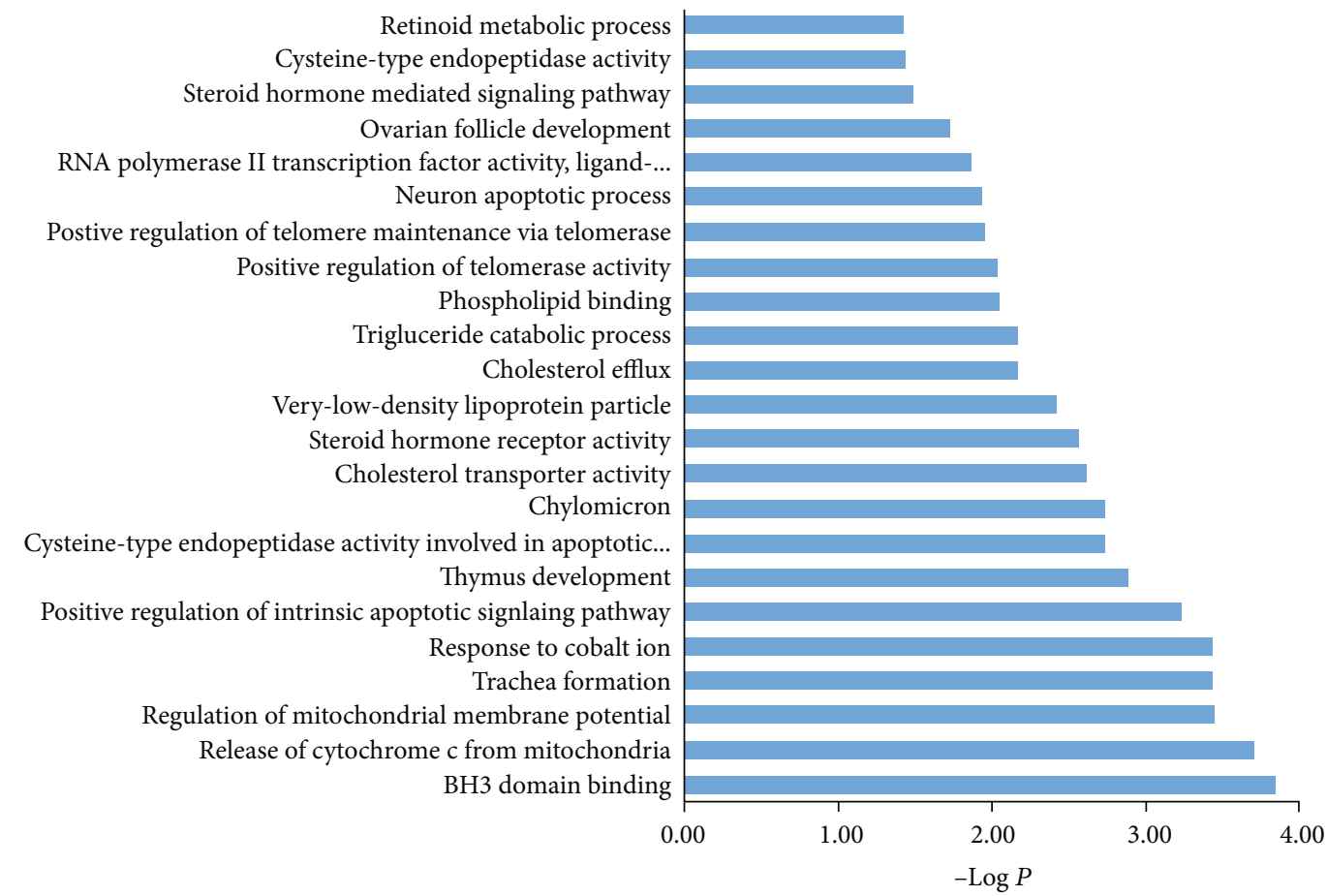

FIGURE 4: GO enrichment analysis of targets. The $y$-axis represents the biological process of the top 23 functional enrichment, and the $x$-axis represents the negative logarithm of $P$ value.

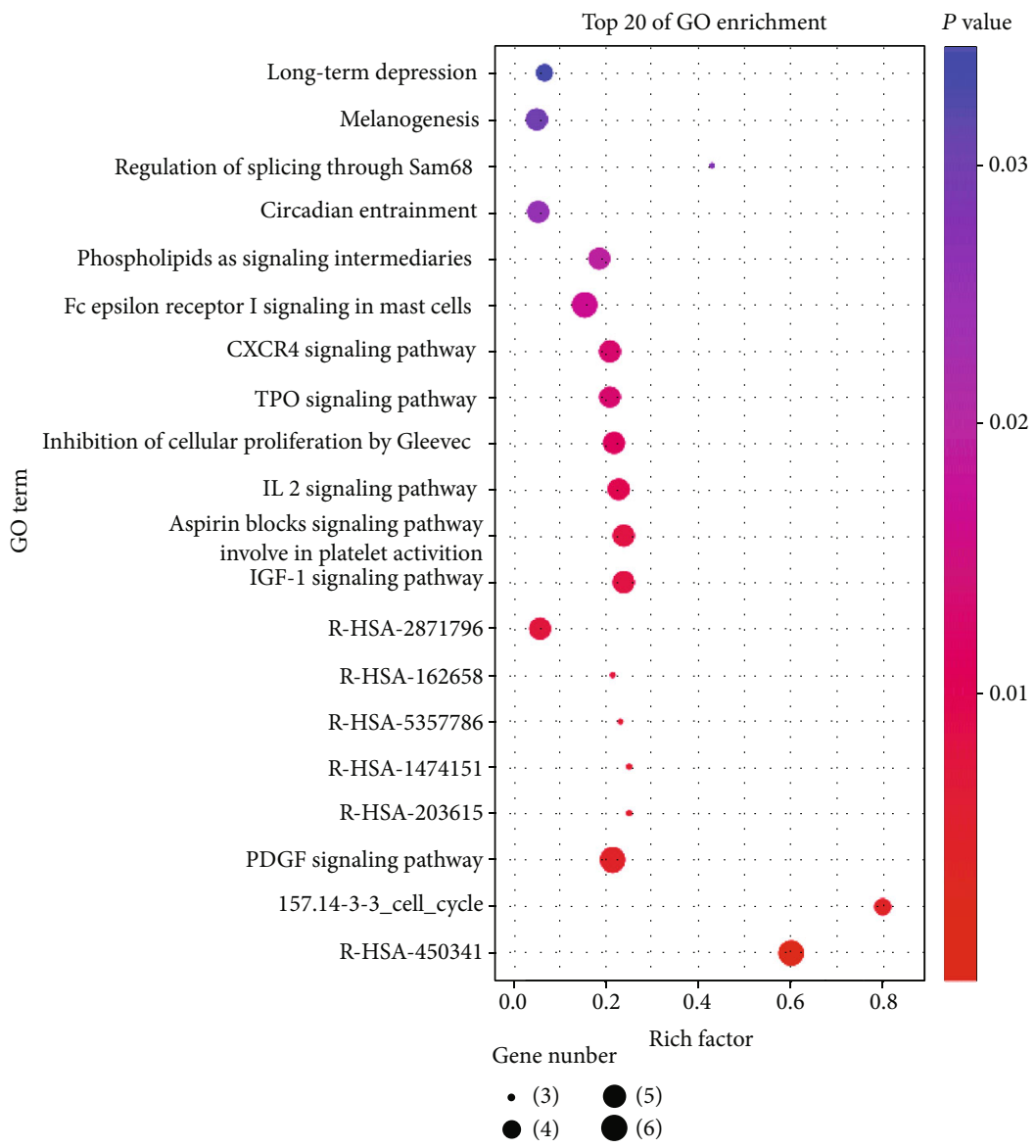

FIGURE 5: Top 20 of pathway enrichment. The circle size stands for gene numbers, the red color and higher rich factor indicate greater enrichment of pathways. 


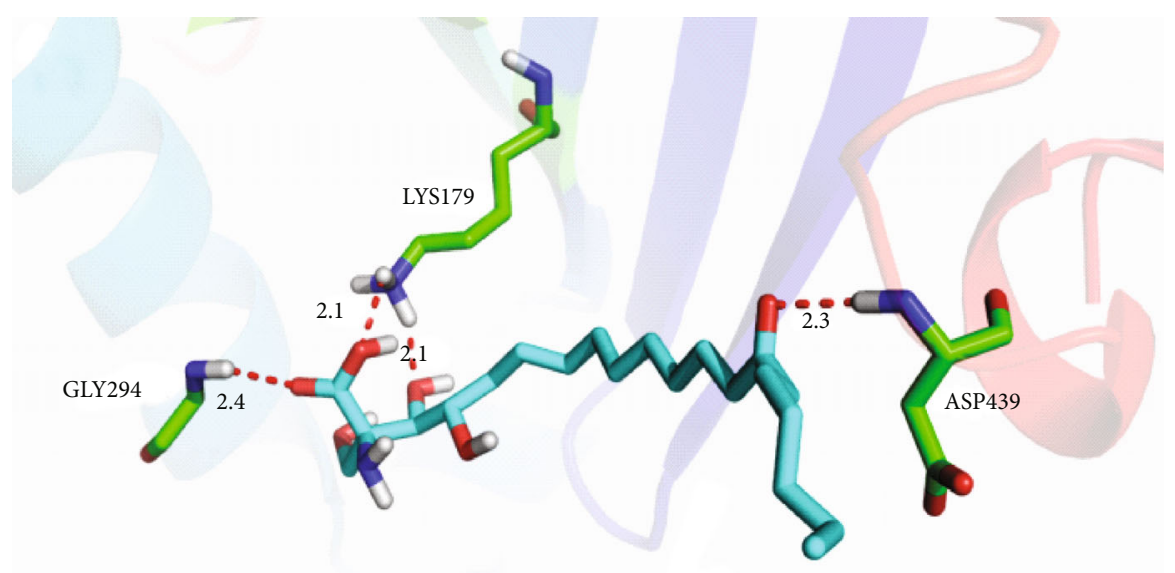

(a)

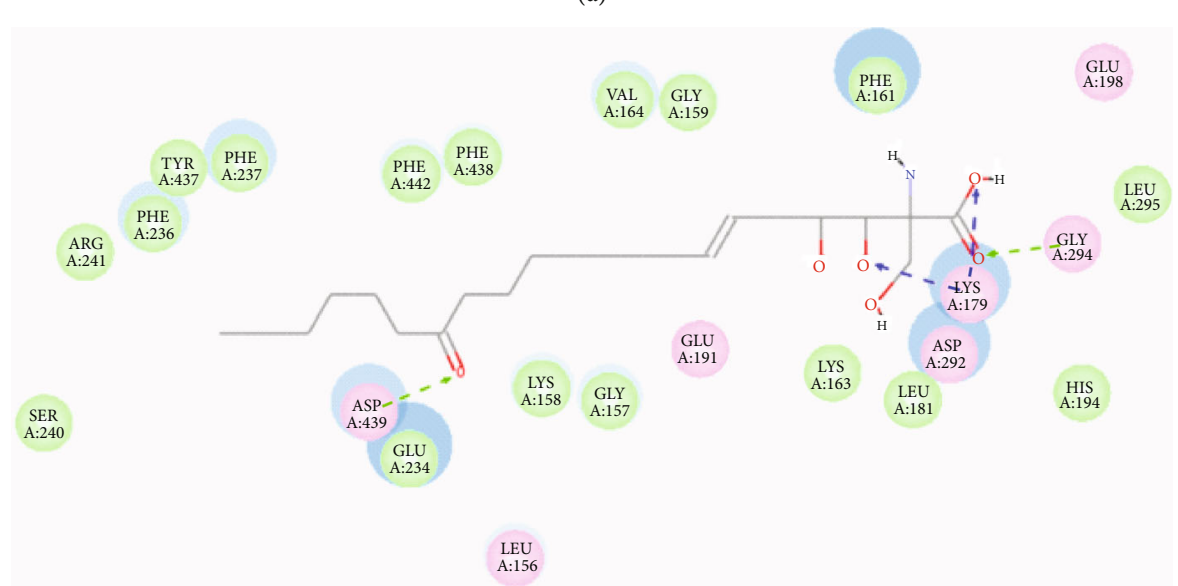

(b)

FIGURE 6: (a) Proposed binding mode of myriocin with AKT1 (PDB code: 3OCB). (b) 2D presentation of interaction between myriocin and AKT1.

The PPI data was obtained by setting the confidence level to 0.7 , and then, the PPI data was imported into the Cytoscape 3.6.0 software to construct the network of protein target interaction (shown in Figure 3). Figure 3 shows a network of 85 interacting proteins with 1466 edges. The average degree of nodes is 34.5. AKT1, MAPK8, TP53, INS, MAPK1, IL6, TNF, JUN, MAPK3, and CASP3 were the targets with higher degree $(>30)$.

3.4. Enrichment Analysis by GO and KEGG. 85 intersection targets were imported into the DAVID database for GO enrichment analysis and pathway enrichment analysis. The GO enrichment analysis is shown in Figure 4. The results of GO enrichment analysis showed that the key intersection targets were mainly concentrated in $\mathrm{BH} 3$ domain binding, release of cytochrome $\mathrm{c}$ from mitochondria, regulation of mitochondrial membrane potential, and other processes. The results of pathway enrichment analysis are shown in Figure 5. Rich factor represented the ratio of the number of genes in the pathway of differentially expressed genes to the total number of target genes in the pathway of all genes.
The larger the rich factor was, the greater the degree of enrichment was. The key targets of this study were mainly enriched in the R-HSA-450341 signaling pathway, 157.14-33 cell cycle signaling pathway, and PDGF signaling pathway.

3.5. Molecular Docking. Molecular docking studies were carried out to investigate the binding modes of myriocin with ATK1 and MAPK8. As shown in Figure 6(a), myriocin bound to AKT1 with four key hydrogen bonds. The carboxylic acid group of myriocin formed two hydrogen bonds with side chain of LYS179 (length: $2.1 \AA$ ) and backbone of GLY294 (length: 2.4 $\mathrm{A}$ ), respectively. One of hydroxyl formed one hydrogen bond with side chain of LYS179 (length: 2.1 $\AA$ ). An additional hydrogen bond (length: 2.1 $\AA$ ) was formed between carbonyl of myriocin and backbone of ASP439. Similarly, myriocin was docked into the binding pocket of MAKP8 through two hydrogen bonds with MET111 (length: $1.7 \AA$ ) and PHE170 (length: 2.1 $\AA$ ), shown in Figure 7(a). Figures 6(b) and 7(b) show the interactions between myriocin and ATK1 and MAPK8, respectively, in 2D diagram. 


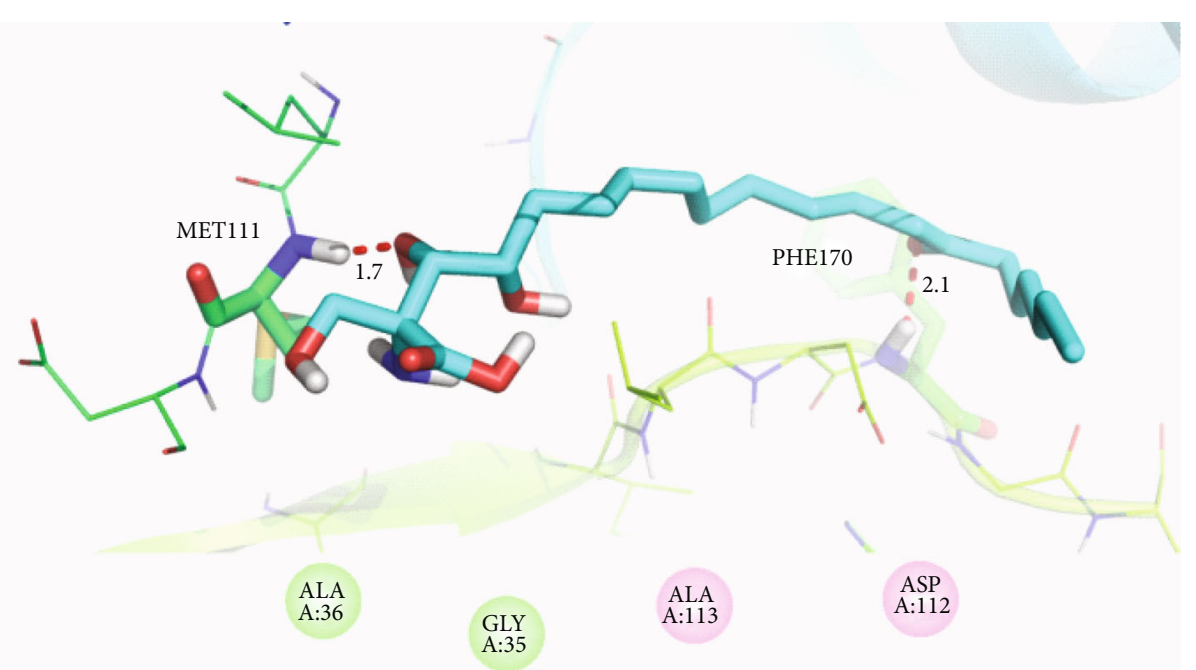

(a)

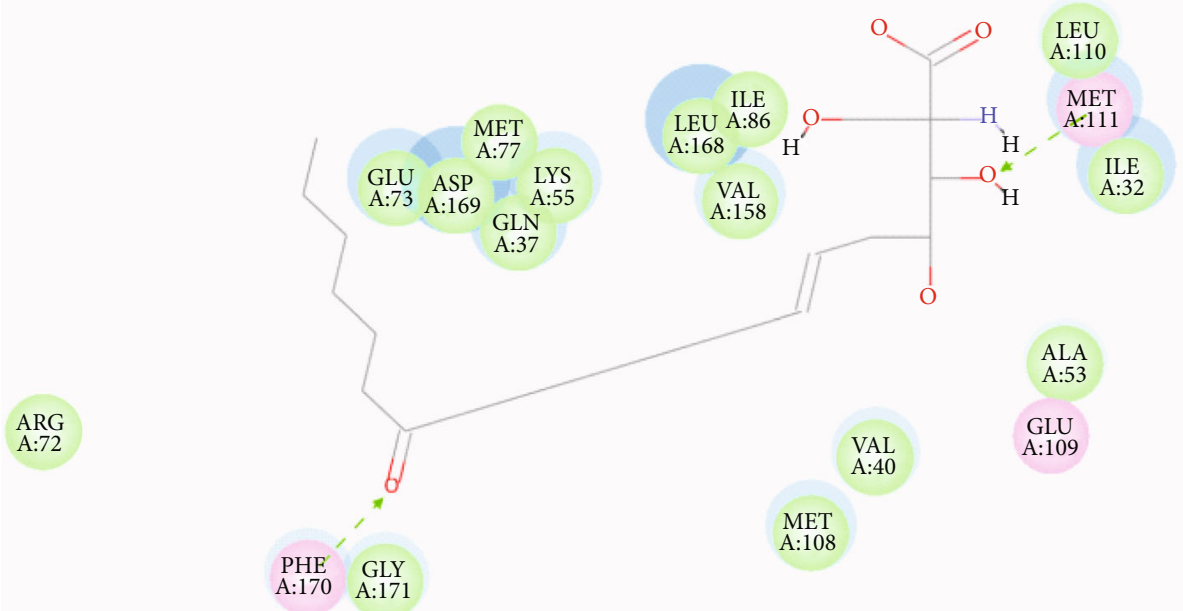

(b)

Figure 7: (a) Proposed binding mode of myriocin with MAPK8 (PDB code: 4G1W). (b) 2D presentation of interaction between myriocin and MAPK8.

\section{Discussion}

Diabetic nephropathy is one of the most frequent microvascular complications in diabetic patients and is the leading cause of end-stage renal disease all over the world [12]. In recent decades, many studies on DN have been carried out, and some results have been achieved. However, no better treatment drugs or measures have been produced so far. In recent years, Chinese traditional medicine in the treatment of DKD has also carried out a lot of researches and has made achievements. Wang et al.'s study has shown that cordyceps sinensis can protect renal tubules and promote renal tubules repair in animal models of drug-induced acute renal injury, thus improving renal function [13]. Researches showed that cordyceps cicadae could alleviate glomerulosclerosis and improve the chronic renal failure [14-16].

This study systematically studied the potential mechanism of cordyceps cicadae for the treatment of DN by the method of network pharmacology and molecular docking analysis and analyzed and constructed the "active ingredient-target-disease" network diagram of cordyceps cicadae for the treatment of DN. 36 main active ingredients of cordyceps cicadae and 85 common targets of DN, including myriocin, guanosine, and adenosine, were screened out through the database search and screening. Besides, key targets such as AKT1, MAPK8, MAPK1, TP53, IL6, and TNF were screened out. Molecular docking studies showed the binding modes of myriocin with ATK1 and MAPK8. AKT is a serine threonine kinase, a central regulator of cell growth, proliferation, survival, and metabolism. Podocyte injury is a predictive indicator of DN. It has been reported that can inhibit podocyte autophagy and aggravate its apoptosis through the AKT pathway [17]. IL-6 is a proinflammatory factor, and one of the important factors in the occurrence of $\mathrm{DN}$ is inflammation. DN patients showed a higher level of IL-6, which positively correlated with the extent of proteinuria [18]. IL-6 seemed to be a good biomarker of chronic kidney injury; the signal transduction 
inflammatory response it participated in was critical to the progression of DN. Study suggests that these IL-6 responses were mediated through a gp130-STAT3-dependent mechanism [19]. TP53 is also called TRIAP1. Under high-glucose conditions, TRIAP1 is a small conserved protein containing 76 amino acids. It is induced by TP53 under low-level genotoxic stress and helps reduce cell death. It has been reported that TRIAP1 interacts with heat shock protein 70 (HSP70) to regulate the apoptosis pathway [20,21]. Studies have shown that TRIAP1 is a direct target of miR-770-5p. The expression of miR-770-5p in podocytes is upregulated under high-glucose conditions, and the downregulation of miR$770-5 \mathrm{p}$ by targeting TRIAP1 can eliminate podocytes induced by high glucose and inhibit cell apoptosis, which further proves that TP53 has a potential role in the occurrence and development of DN [22]. In a diabetic environment, the increasing production of glycation end products activates NF- $\kappa \mathrm{B}, \mathrm{MAPK}$, and other signaling pathways, which in turn mediates the activation of TGF- $\beta$ signaling pathways and promotes the synthesis and deposition of extracellular matrix [23]. MAPK8 is related to lipid metabolism [24], and lipid metabolism disorder is an important risk factor for the occurrence of DN. GO enrichment analysis results showed that the key targets were mainly enriched in $\mathrm{BH} 3$ domain binding, release of cytochrome $\mathrm{c}$ from mitochondria, and regulation of mitochondrial membrane potential biological processes. It was reported in the literature that the $\mathrm{BH} 3$ domain was involved in the process of cell apoptosis and autophagy [25]. Mitochondria also play a very important role in the process of cell apoptosis [26]. The results of pathway enrichment indicated that RHSA-450341, 157.14-3-3 cell cycle, and PDGF signal pathways may be related to the treatment of DN by cordyceps sinensis. Among the active compounds of cordyceps cicadae, the candidate compounds that may be related to these targets include myriocin, $\beta$-sitosterol, guanosine, arachidonic acid, inosine, berberine, cinnamaldehyde, linoleyl acetate, uralene, linoleic acid, and deoxyandrographolide. Myriocin (ISP-1, thermozymocidin), an atypical amino acid, was isolated from the culture broth, mycelia, and sporoderm-broken spore powders of cordyceps cicadae [27]. The molecular docking studies showed that myriocin bound to AKT1 with four key hydrogen bonds as well as to MAKP8 through two hydrogen bonds. The results revealed that myriocin, one active compound of cordyceps cicadae, may act on AKT1 and MAPK8. However, the main active compounds of cordyceps cicadae such as myriocin that may act on the DN targets require further experimental verifications and explorations. The interaction of active compounds from cordyceps cicadae needs further research. In order to verify the effectiveness of network pharmacological screening of active compounds and targets, we will further use molecular biology methods to investigate the effects and molecular mechanism of candidate compounds on targets and their effects on DN. These potential mechanisms for the treatment of DN by cordyceps cicadae will provide good ideas and directions for the next step of experimental verification and innovative drug development.
In conclusion, our study predicted target proteins related to candidate compounds of cordyceps cicadae by network pharmacology and verified by molecular docking. Since traditional Chinese medicine contains a variety of active compounds, they can act on multiple targets and signal pathways at the same time and produce a synergistic effect, which can be an effective method for the treatment of DN. Moreover, we can find particularly active compounds of traditional Chinese medicine. It is possible to develop innovative drugs for the treatment of $\mathrm{DN}$ diseases and to break through the difficult problems in the treatment of DN.

\section{Data Availability}

The data used to support the findings of this study are available from the corresponding author upon request.

\section{Conflicts of Interest}

The authors declare that there is no conflict of interests regarding the publication of this paper.

\section{Authors' Contributions}

X. S. and ZJ. P. contributed to the conception and design of the study. Y. Q., X. W., and X. Y. contributed to the conduct/data collection. Y. Q. contributed to data analysis. Y. Q. and X. W. contributed to manuscript writing. Yi Qian and Xin Sun contributed equally to this work.

\section{Acknowledgments}

This work was supported by the Science, Education and Health Engineering-Medical Innovation Team Project of Wuxi (grant number CXTD007), Jiangsu Pharmaceutical Association Osaikang Fund Project (grant number A2017033), General Program of Science and Technology Plan of Wuxi Municipal Health Commission (grant number MS201708), Primary Research and Development Plan Programs of Jiangsu Province (grant number 2019633), Science and Technology Development Fund Project of Nanjing Medical University (grant number NMUB2020277), and Translational Medicine Research Project for Translational Medicine Center of Wuxi (grant number 2020ZHYB11).

\section{References}

[1] R. Z. Alicic, M. T. Rooney, and K. R. Tuttle, "Diabetic kidney disease," Clinical Journal of the American Society of Nephrology, vol. 12, no. 12, pp. 2032-2045, 2017.

[2] M. Afkarian, M. C. Sachs, B. Kestenbaum et al., "Kidney disease and increased mortality risk in type 2 diabetes," Journal of the American Society of Nephrology, vol. 24, no. 2, pp. 302-308, 2013.

[3] M. C. Thomas, M. Brownlee, K. Susztak et al., "Diabetic kidney disease," Nature Reviews Disease Primers, vol. 1, no. 1, pp. 120, 2015.

[4] B. Pang, L. H. Zhao, Q. Zhou et al., "Application of berberine on treating type 2 diabetes mellitus," International Journal of Endocrinology, vol. 2015, 12 pages, 2015. 
[5] L. Xiang, P. Jiang, L. Zhou et al., "Additive effect of Qidan Dihuang grain, a traditional Chinese medicine, and angiotensin receptor blockers on albuminuria levels in patients with diabetic nephropathy: a randomized, parallel-controlled trial," Evidence-based Complementary and Alternative Medicine, vol. 2016, 8 pages, 2016.

[6] I. M. Liu, W. Fu, W. Yi, Z. Xu, and N. Xu, "The Neural Pathway of Reflex Regulation of Electroacupuncture at Orofacial Acupoints on Gastric Functions in Rats," Evidence-based Complementary and Alternative Medicine, vol. 2012, 8 pages, 2012.

[7] W.-B. Zeng, H. Yu, F. Ge et al., "Distribution of nucleosides in populations of cordyceps cicadae," Molecules, vol. 19, no. 5, pp. 6123-6141, 2014.

[8] I. C. Li, S. Lin, Y. T. Tsai et al., "Cordyceps cicadae mycelia and its active compound HEA exert beneficial effects on blood glucose in type 2 diabetic $\mathrm{db} / \mathrm{db}$ mice," Journal of the Science of Food and Agriculture, vol. 99, no. 2, pp. 606-612, 2019.

[9] Q. Zhang, O. J. Olatunji, H. Chen, A. J. Tola, and O. O. Oluwaniyi, "Evaluation of the anti-diabetic activity of polysaccharide from cordyceps cicadae in experimental diabetic rats," Chemistry \& Biodiversity, vol. 15, no. 8, article e1800219, 2018.

[10] J. Yang, H. Dong, Y. Wang et al., "Cordyceps cicadae polysaccharides ameliorated renal interstitial fibrosis in diabetic nephropathy rats by repressing inflammation and modulating gut microbiota dysbiosis," International Journal of Biological Macromolecules, vol. 163, pp. 442-456, 2020.

[11] H. Yuan, Q. Ma, H. Cui et al., "How can synergism of traditional medicines benefit from network pharmacology?," Molecules, vol. 22, no. 7, p. 1135, 2017.

[12] D. Sanajou, A. Ghorbani Haghjo, H. Argani, and S. Aslani, "AGE-RAGE axis blockade in diabetic nephropathy: current status and future directions," European Journal of Pharmacology, vol. 833, pp. 158-164, 2018.

[13] H.-P. Wang, C. W. Liu, H. W. Chang, J. W. Tsai, Y. Z. Sung, and L. C. Chang, "Cordyceps sinensis protects against renal ischemia/reperfusion injury in rats," Molecular Biology Reports, vol. 40, no. 3, pp. 2347-2355, 2013.

[14] H. Wang, J. Zhang, W. H. Sit, C. Y. Lee, and J. M. F. Wan, "Cordyceps cicadae induces G2/M cell cycle arrest in MHCC97H human hepatocellular carcinoma cells: a proteomic study," Chinese Medicine, vol. 9, no. 1, p. 15, 2014.

[15] R. Zhu, Y. P. Chen, Y. Y. Deng et al., "Cordyceps cicadae extracts ameliorate renal malfunction in a remnant kidney model," Journal of Zhejiang University SCIENCE B, vol. 12, no. 12, pp. 1024-1033, 2011.

[16] J.-H. Hsu, B. Y. Jhou, S. H. Yeh, and Y. L. Chen, "Healthcare functions of cordyceps cicadae," Journal of Nutrition \& Food Sciences, vol. 5, no. 6, 2015.

[17] J. Xu, Y. Deng, Y. Wang, X. Sun, S. Chen, and G. Fu, "SPAG5AS1 inhibited autophagy and aggravated apoptosis of podocytes via SPAG5/AKT/mTOR pathway," Cell Proliferation, vol. 53, no. 2, p. e12738, 2020.

[18] M. Dalla Vestra, M. Mussap, P. Gallina et al., “Acute-phase markers of inflammation and glomerular structure in patients with type 2 diabetes," Journal of the American Society of Nephrology, vol. 16, 3 suppl 1, pp. S78-S82, 2005.

[19] E. Feigerlová and S.-F. Battaglia-Hsu, "IL-6 signaling in diabetic nephropathy: from pathophysiology to therapeutic perspectives," Cytokine \& Growth Factor Reviews, vol. 37, pp. 57-65, 2017.
[20] T. Riley, E. Sontag, P. Chen, and A. Levine, "Transcriptional control of human p53-regulated genes," Nature Reviews Molecular Cell Biology, vol. 9, no. 5, pp. 402-412, 2008.

[21] W. R. Park and Y. Nakamura, "p53CSV, a novel p53-inducible gene involved in the p53-dependent cell-survival pathway," Cancer Research, vol. 65, no. 4, pp. 1197-1206, 2005.

[22] S.-Z. Zhang, X. J. Qiu, S.-S. Dong et al., "MicroRNA-770-5p is involved in the development of diabetic nephropathy through regulating podocyte apoptosis by targeting TP53 regulated inhibitor of apoptosis 1," European Review for Medical and Pharmacological Sciences, vol. 23, no. 3, pp. 1248-1256, 2019.

[23] V. Vallon and R. Komers, "Pathophysiology of the diabetic kidney," Comprehensive Physiology, vol. 1, no. 3, pp. 1175$1232,2011$.

[24] X. J. Huang, C. J. He, S. Liang et al., "Veratrilla baillonii Franch could alleviate lipid accumulation in $\mathrm{LO} 2$ cells by regulating oxidative, inflammatory, and lipid metabolic signaling pathways," Frontiers in Pharmacology, vol. 11, p. 11, 2020.

[25] C. W. Chu, M.-. C. Yang, C.-. H. Chou et al., "GSK3 $\beta$-mediated Ser156 phosphorylation modulates a $\mathrm{BH} 3$-like domain in BCL2L12 during TMZ-induced apoptosis and autophagy in glioma cells," International Journal of Molecular Medicine, vol. 42, pp. 905-918, 2018.

[26] M. Zhang, J. Zheng, R. Nussinov, and B. Ma, "Release of cytochrome C from Bax pores at the mitochondrial membrane," Scientific Reports, vol. 7, no. 1, article 2825, 2017.

[27] X. Zhang, Q. Hu, and Q. Weng, "Secondary metabolites (SMs) ofIsaria cicadaeandIsaria tenuipes," RSC Advances, vol. 9, no. 1, pp. 172-184, 2019. 